\title{
Jordan normal form projections
}

\author{
By
}

KeE YUEN LaM, ANDREW RANICKI and LARry SMITH

Let $K$ be a field, and let $T: V \rightarrow V$ be an endomorphism of a finite-dimensional vector space $V$ over $K$ such that $K$ contains the roots $\lambda_{1}, \lambda_{2}, \ldots, \lambda_{m}$ of the characteristic polynomial

$$
\chi(t)=\operatorname{det}(t I-T)=\left(t-\lambda_{1}\right)^{n_{1}}\left(t-\lambda_{2}\right)^{n_{2}} \cdots\left(t-\lambda_{m}\right)^{n_{m}} .
$$

$V$ is a direct sum of $T$-invariant subspaces $V_{j}=\operatorname{ker}\left(T-\lambda_{j} I\right)^{n_{j}}$, one for each eigenvalue $\lambda_{j}$, such that $T-\lambda_{j} I: V_{k} \rightarrow V_{k}$ is nilpotent for $j=k$ and an automorphism for $j \neq k$. We obtain in this paper an explicit formula for the projection $p_{j}(T)=p_{j}(T)^{2}: V \rightarrow V$ onto the subspace $V_{j}$, as a polynomial in $T$.

A near-projection in a ring $A$ is an element $p \in A$ such that $q=p(1-p) \in A$ is nilpotent, that is $q^{n}=0$ for some exponent $n \geqq 1$. We refer to Lück and Ranicki [3] for the general theory of near-projections, and for the construction of the unique projection $p_{\omega}=\left(p_{\omega}\right)^{2} \in A$ such that $p_{\omega}-p$ is nilpotent and $p p_{\omega}=p_{\omega} p$, namely

$$
\begin{aligned}
p_{\omega}= & \left(p^{n}+(1-p)^{n}\right)^{-1} p^{n}=p+(1 / 2)(2 p-1)\left((1-4 q)^{-1 / 2}-1\right) \\
= & p+(2 p-1)\left(q+3 q^{2}+10 q^{3}+35 q^{4}+126 q^{5}+462 q^{6}+1716 q^{7}\right. \\
& +6435 q^{8}+24310 q^{9}+92378 q^{10}+352716 q^{11} \\
& +1352078 q^{12}+\cdots \in A .
\end{aligned}
$$

(In the special case when $A$ is of characteristic 2 this simplifies to

$$
\left.p_{\omega}=p+q+q^{2}+q^{4}+q^{8}+q^{16}+\cdots \in A\right) \text {. }
$$

If $R$ is any ring and $p: V \rightarrow V$ is a near-projection in the endomorphism ring of an $R$-module $V$ then $p_{\omega}: V \rightarrow V$ is the projection onto the $p$-invariant submodule

$$
P=\operatorname{im}\left(\mathrm{p}_{\omega}\right)=\operatorname{im}\left(p^{n}\right)=\operatorname{ker}\left((1-p)^{n}\right) \subseteq V,
$$

such that $V=P \oplus Q$ with $Q$ the $p$-invariant submodule

$$
Q=\operatorname{im}\left(1-p_{\omega}\right)=\operatorname{im}\left((1-p)^{n}\right)=\operatorname{ker}\left(p^{n}\right) \subseteq V .
$$

$p: P \rightarrow P$ is an isomorphism and $p: Q \rightarrow Q$ is nilpotent. An endomorphism $p: V \rightarrow V$ of a finite-dimensional vector space $V$ over a field $K$ is a near-projection if and only if the characteristic polynomial is of the type $\chi(t)=t^{m}(1-t)^{n}$, in which case $(p(1-p))^{\max (m, n)}$ $=0: V \rightarrow V$. 
Let $R$ be a commutative ring with 1 , with polynomial extension ring $R[t]$. A polynomial $\varphi(t) \in R[t]$ is completely reducible if it can be expressed as a product of linear factors

$$
\varphi(t)=\left(t-\lambda_{1}\right)^{n_{1}}\left(t-\lambda_{2}\right)^{n_{2}} \cdots\left(t-\lambda_{m}\right)^{n_{m}} \in R[t] .
$$

Let $T: V \rightarrow V$ be an endomorphism of an $R$-module $V$. A polynomial $\varphi(t) \in R[t]$ annihilates $T$ if

$$
\varphi(T)=0: V \rightarrow V,
$$

in which case $V$ is an $R[t] /(\varphi(t))$-module with $t$ acting on $V$ by $T$. Any expression of $R[t] /(\varphi(t))$ as a product of rings

$$
R[t] /(\varphi(t))=A_{1} \times A_{2} \times \cdots \times A_{m}
$$

determines a decomposition of $V$ as a direct sum of $T$-invariant submodules

$$
V=V_{1} \oplus V_{2} \oplus \cdots \oplus V_{m},
$$

with $V_{j}=A_{j} V$ an $A_{j}$-module.

The characteristic polynomial of an endomorphism $T: V \rightarrow V$ of a finitely generated free $R$-module $V$ is defined by

$$
\chi(t)=\operatorname{det}((t I-T): V[t] \rightarrow V[t]) \in R[t],
$$

as usual. $\chi(t)$ annihilates $T$ by the Cayley-Hamilton theorem. More generally, Amkvist [1] defined the characteristic polynomial of an endomorphism $T: V \rightarrow V$ of a finitely generated projective $R$-module $V$ by

$$
\chi(t)=t^{n} \lambda(-1 / t) \in R[t],
$$

with $n$ the degree of the polynomial

$$
\lambda(t)=\operatorname{det}(I+t(T \oplus 0):(V \oplus W)[t] \rightarrow(V \oplus W)[t]) \in R[t]
$$

defined for any finitely generated projective $R$-module $W$ such that $V \oplus W$ is a finitely generated free $R$-module. This agrees with the previous definition, and is also such that $\chi(T)=0: V \rightarrow V$.

Theorem. Let $T: V \rightarrow V$ be an endomorphism of an $R$-module $V$ such that there exists $a$ completely reducible annihilating polynomial of degree $n$

$$
\varphi(t)=\left(t-\lambda_{1}\right)^{n_{1}}\left(t-\lambda_{2}\right)^{n_{2}} \cdots\left(t-\lambda_{m}\right)^{n_{m}} \in R[t] \quad\left(n=\sum_{j=1}^{m} n_{j}\right),
$$

with the eigenvalues $\lambda_{1}, \lambda_{2}, \ldots, \lambda_{m} \in R$ such that each $\lambda_{j}-\lambda_{k} \in R(j \neq k)$ is a unit. For $j=1,2, \ldots, m$ define the polynomial of degree $\left(n-n_{j}\right)$

$$
g_{j}(t)=\prod_{k \neq j}\left(t-\lambda_{k}\right)^{n_{k}} \in R[t],
$$

so that $\varphi(t)=\left(t-\lambda_{j}\right)^{n_{j}} g_{j}(t)$. Then

$$
g_{j}\left(\lambda_{j}\right)^{-1} g_{j}(T): V \rightarrow V
$$


is a near-projection in the endomorphism ring of $V$, and its associated projection

$$
p_{j}(T)=\left(g_{j}\left(\lambda_{j}\right)^{-1} g_{j}(T)\right)_{\omega}: V \rightarrow V
$$

is the projection onto the direct summand

with

$$
V_{j}=\operatorname{im}\left(g_{j}(T): V \rightarrow V\right)=\operatorname{ker}\left(\left(T-\lambda_{j} I\right)^{n_{j}}: V \rightarrow V\right) \subseteq V,
$$

$$
\sum_{j=1}^{m} p_{j}(T)=1: V \rightarrow V, \quad p_{j}(T) p_{k}(T)=0 \quad \text { for } j \neq k
$$

$V$ is the direct sum of the T-invariant submodules $V_{j}(j=1,2, \ldots, m)$, such that $T-\lambda_{j} I: V_{k} \rightarrow V_{k}$ is nilpotent for $j=k$ and an automorphism for $j \neq k$.

Proof. For $j=1,2, \ldots, m$ define

$$
a_{j}(t)=\sum_{k=0}^{n_{j}-1} \frac{\left(t-\lambda_{j}\right)^{k}}{k !}\left(\frac{1}{g_{j}(t)}\right)_{t=\lambda_{j}}^{(k)} \in R[t],
$$

the first $n_{j}$ terms in the Taylor expansion of $1 / g_{j}(t)$ around $t=\lambda_{j}$, such that

$$
a_{1}(t) g_{1}(t)+a_{2}(t) g_{2}(t)+\cdots+a_{m}(t) g_{m}(t)=1 \in R[t] .
$$

The left hand side is the degree $\left(n_{j}-1\right)$ Lagrange-Sylvester interpolation polynomial $f(t)$ with $f\left(\lambda_{j}\right)=1, f^{(k)}\left(\lambda_{j}\right)=0\left(1 \leqq j \leqq m, 1 \leqq k \leqq n_{j}-1\right)$ (Gantmacher [2, V.1]). The coefficients of $a_{j}(t)$ are defined in $R$, since the coefficients of the formal power series

$$
\begin{aligned}
\left(t-\lambda_{i}\right)^{-n_{i}} & =\left(\lambda_{j}-\lambda_{i}\right)^{-n_{i}}\left(1+\left(t-\lambda_{j}\right) /\left(\lambda_{j}-\lambda_{i}\right)\right)^{-n_{i}} \\
& =\sum_{k=0}^{\infty}(-1)^{k}\left(\begin{array}{c}
n_{i}+k-1 \\
k
\end{array}\right)\left(t-\lambda_{j}\right)^{k} /\left(\lambda_{j}-\lambda_{i}\right)^{k-n_{i}} \in R\left[\left[t-\lambda_{j}\right]\right]
\end{aligned}
$$

are defined in $R$, for $i \neq j, \varphi(t)$ is a factor of $g_{j}(t) g_{k}(t)$ for $j \neq k$, so that there are defined projections

$$
p_{j}(t)=a_{j}(t) g_{j}(t) \in R[t] /(\varphi(t)) \quad(1 \leqq j \leqq m)
$$

such that

$$
\sum_{j=1}^{m} p_{j}(t)=1, \quad p_{j}(t) p_{k}(t)=0 \quad \text { for } \quad j \neq k
$$

The isomorphism of rings defined by projection on each factor

$$
R[t] /(\varphi(t)) \rightarrow \prod_{j=1}^{m} R[t] /\left(\left(t-\lambda_{j}\right)^{n_{j}}\right): x \rightarrow(x, x, \ldots, x)
$$

has inverse

$$
\begin{aligned}
\prod_{j=1}^{m} R[t] /\left(\left(t-\lambda_{j j}\right)^{n_{j}}\right) & \rightarrow R[t] /(\varphi(t)) ; \\
\quad\left(x_{1}, x_{2}, \ldots, x_{m}\right) & \rightarrow x_{1} p_{1}(t)+x_{2} p_{2}(t)+\cdots+x_{m} p_{m}(t) .
\end{aligned}
$$


The elements $g_{j}(t),\left(t-\lambda_{j}\right)^{n_{j}} \in R[t] /(\varphi(t))$ are coprime and have product 0 , so that $\operatorname{in}\left(g_{j}(T): V \rightarrow V\right)=\operatorname{ker}\left(\left(T-\lambda_{j} I\right)^{n_{j}}: V \rightarrow V\right)$.

Let $V_{j} \leqq V$ be the image of the projection

$$
p_{j}(T)=a_{j}(T) g_{j}(T): V \rightarrow V,
$$

an $R[t] /\left(\left(t-\lambda_{j}\right)^{n_{j}}\right)$-module with $t$ acting by $T: V_{j} \rightarrow V_{j}$, and with $g_{j}(T), a_{j}(T): V_{j} \rightarrow V_{j}$ inverse automorphisms. Thus

$$
V_{j}=\operatorname{im}\left(p_{j}(T): V \rightarrow V\right)=\operatorname{im}\left(g_{j}(T): V \rightarrow V\right)=\operatorname{ker}\left(\left(T-\lambda_{j} I\right)^{\mathrm{n}_{j}}: V \rightarrow V\right) .
$$

As $\lambda_{j}$ is a root of $g_{j}(t)-g_{j}\left(\lambda_{j}\right)$ there exists a polynomial $h_{j}(t)$ such that

$$
g_{j}(t)-g_{j}\left(\lambda_{j}\right)=\left(t-\lambda_{j}\right) h_{j}(t) \in R[t] .
$$

Similarly, there exists a polynomial $b_{j}(t)$ such that

Now

$$
a_{j}(t)-a_{j}\left(\lambda_{j}\right)=\left(t-\lambda_{j}\right) b_{j}(t) \in R[t]
$$

$$
a_{j}\left(\lambda_{j}\right) g_{j}(t)=g_{j}\left(\lambda_{j}\right)^{-1} g_{j}(t) \in R[t] /(\varphi(t))
$$

is a near-projection, since

$$
g_{j}\left(\lambda_{j}\right)^{-1} g_{j}(t)\left(1-g_{j}\left(\lambda_{j}\right)^{-1} g_{j}(t)\right)=-g_{j}\left(\lambda_{j}\right)^{-2} g_{j}(t)\left(t-\lambda_{j}\right) h_{j}(t) \in R[t] /(\varphi(t))
$$

is nilpotent (of exponent at most $n_{j}$ ). The near-projection differs from the projection $p_{j}(t)$ by a nilpotent

so that

$$
p_{j}(t)-g_{j}\left(\lambda_{j}\right)^{-1} g_{j}(t)=g_{j}(t)\left(t-\lambda_{j}\right) b_{j}(t) \in R[t] /(\varphi(t)),
$$

$$
p_{j}(t)=\left(g_{j}\left(\lambda_{j}\right)^{-1} g_{j}(t)\right)_{\omega} \in R[t] /(\varphi(t))
$$

$\mathrm{R}$ e $\mathrm{m}$ a $\mathrm{k} \mathrm{k}$. The hypotheses of the Theorem are satisfied for any endomorphism $T: V \rightarrow V$ of a finite-dimensional vector space $V$ over a field $K$ which contains the roots of the minimal polynomial $\mu(t)$, with $\varphi(t)$ either $\mu(t)$ or the characteristic polynomial $\chi(t)$. The near-projections $g_{j}\left(\lambda_{j}\right)^{-1} g_{j}(t): V \rightarrow V$ for the two cases need not be the same, but they differ by nilpotents so that the projections $p_{j}(T)=\left(g_{j}\left(\lambda_{j}\right)^{-1} g_{j}(T)\right)_{\omega}: V \rightarrow V$ are the same.

Remark 2. The projections $p_{j}(T)$ can also be expressed as

$$
p_{j}(T)=\left(\left(T-\lambda_{j} I\right)^{n_{j}}+g_{j}(T)\right)^{-1} g_{j}(T): V \rightarrow V
$$

R e m a rk 3. The proof of the Theorem does not actually use the Jordan normal form, and could be used to simplify the standard proofs. We are indepted to S. J. Patterson for describing to us Fitting's proof, making use of the projections $p_{j}(T)$ but without an explicit formula. 


\section{References}

[1] G. Almkvist, Endomorphisms of finitely generated projective modules over a commutative ring. Ark. Math. 11, 263-301 (1974).

[2] F. R. GantmaCher, Matrizenrechnung I. Allgemeine Theorie. Berlin 1958.

[3] W. LÜCK and A. RANICKI, Chain homotopy projections. Preprint 1986.

Eingegangen am 21.11. 1986

Anschriften der Autoren:

Larry Smith

Mathematisches Institut der Georg-August-Universität

Bunsenstr. 3-5

D-3400 Göttingen
Kee Yuen Lam

Department of Mathematics University of British Columbia

Vancouver V6T 1 Y4

British Columbia, Canada
Andrew Ranicki

Department of Mathematics

Univeristy of Edinburgh

Edinburgh, Scotland 